Supporting information

\title{
Heavy metals mediated phospholipids scrambling by human phospholipid scramblase 3: a probable role in mitochondrial apoptosis
}

Santosh Kumar Palanirajan and Sathyanarayana N Gummadi*

From the Applied and Industrial Microbiology Laboratory, Department of Biotechnology,

Bhupat and Jyoti Mehta School of Biosciences, Indian Institute of Technology Madras,

Chennai 600 036, India

*To whom correspondence should be addressed:

Tel: (+91)-44-2257-4114; Fax: (+91)-44-2257-4102; E-mail: gummadi@iitm.ac.in 
Table of Contents

Materials and Methods$-\mathbf{S - 3}$

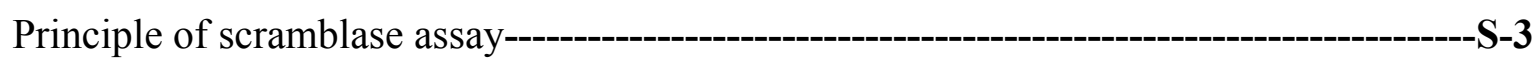

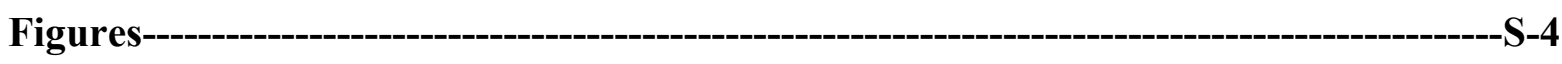

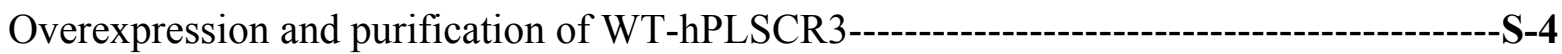




\section{Materials and methods}

\section{Principle of scramblase assay}

Inside labeling: Inside labeled proteoliposomes were generated by reconstituting the NBD labeled lipids along with other phospholipids and protein. Once the proteoliposomes were generated the NBD labeled lipids in the outer leaflet was quenched with the membraneimpermeable dithionite quencher. Therefore in case of inside labeled proteoliposomes, the NBD labeled lipids are present only in the inner leaflet of the vesicle. The hPLSCR3 protein reconstituted in the proteoliposomes will orient in such a way that only $50 \%$ of the protein added will be reconstituted with appropriate orientation such that the $\mathrm{Ca}^{2+}$ binding motif is exposed for binding. In proteoliposomes incubated with $\mathrm{Ca}^{2+}$, the hPLSCR3 is activated and disrupts the asymmetry of the bilayer by translocation of the phospholipids to the outer leaflet which is then quenched by further addition of dithionite and the residual fluorescence is measured after addition. In Proteoliposomes incubated with EDTA, the hPLSCR3 is not activated and hence there will be no translocation of labeled lipids from inner leaflet. The NBD labeled lipids in the outer leaflet already quenched, the NBD labeled lipids in the inner leaflet contributes for the residual fluorescence and the intensity is higher than that of the $\mathrm{Ca}^{2+}$ treated sample (Figure 1). The measure in the difference of residual fluorescence intensity gives the activity of scramblase.

Outside labeling: Proteoliposomes were generated by reconstituting the phospholipids and protein followed by labeling. The NBD labeled phospholipids were added to the proteoliposomes and incubated resulting in outside labeled vesicles. In Proteoliposomes incubated with EDTA, the hPLSCR3 is not activated and translocation of phospholipids does not occur and the NBD labeled lipids are quenched on dithionite addition whereas, in $\mathrm{Ca}^{2+}$ treated sample the hPLSCR3 is activated and the translocation of phospholipids happens from outer leaflet to inner leaflet of the liposomes. This sequestration from quencher retains the residual fluorescence in case of $\mathrm{Ca}^{2+}$ treated samples. The difference in residual fluorescence intensity gives the scramblase activity (Figure 1). 


\section{Figures}

A

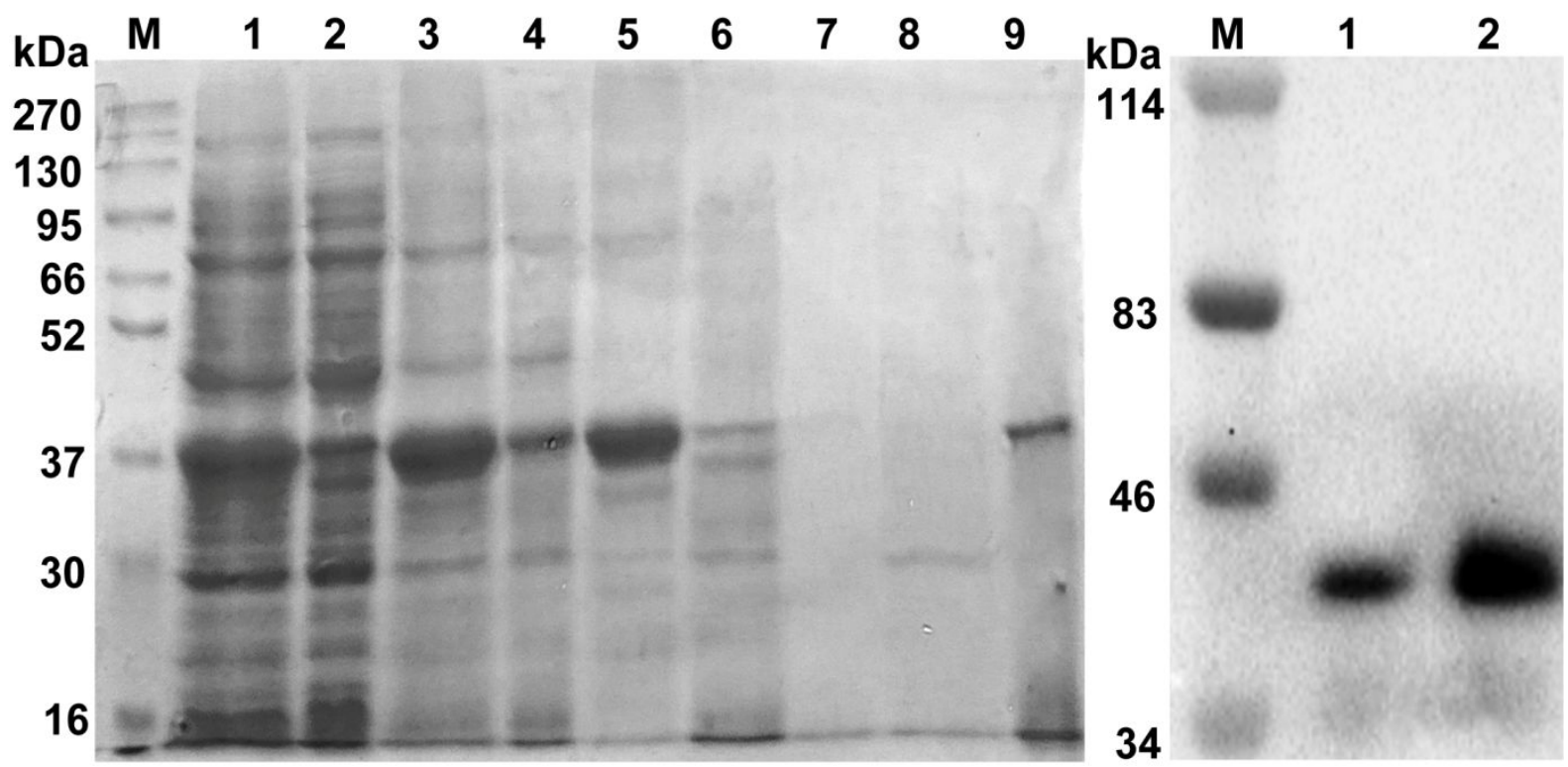

Figure S1. (A) Overexpression and purification of WT-hPLSCR3. Cell-lysate and protein fractions purified by immobilized metal affinity chromatography were visualized in $12 \%$ SDS PAGE. Lane M-Marker, Lane 1 - Induced cell lysate, Lane 2- Soluble fraction after cell lysis, Lane 3- Insoluble fraction after cell lysis (inclusion bodies-IBs), Lane 4- Insoluble fraction (after NLS treatment of IBs), Lane 5-Soluble fraction (after NLS treatment of IBs), Lane 6- Flow-through, Lane 7-wash (elution with $20 \mathrm{mM}$ Imidazole from $\mathrm{Ni}^{2+}$-NTA column), Lane 8-wash (elution with $50 \mathrm{mM}$ Imidazole from $\mathrm{Ni}^{2+}$-NTA column) Lane 9 -Purified WT-hPLSCR3 (elution with $250 \mathrm{mM}$ Imidazole from $\mathrm{Ni}^{2+}$-NTA column). (B) Western Blot analysis of purified WT-hPLSCR3. Lane M-Marker, Lane 1- Induced cell lysate Lane 2purified WT-hPLSCR3. 